\title{
Effect of User Distribution on the Capacity of Cellular Networks
}

\author{
Xue Tang \\ School of Information and Communication Engineering \\ Beijing University of Posts and Telecommunications \\ Beijing, China, +8613581950645 \\ xue.tang@hotmail.com
}

\begin{abstract}
User distribution is a critical issue in cellular networks. In most previous works, a uniform user distribution is often assumed within a cell. In this paper, we purpose a non-uniform user distribution model. Both its effects on downlink and uplink capacities of a cellular network are evaluated under two common packet scheduling algorithms: Maximum Carrier to Interference scheduling and Round Robin scheduling. Simulation results show that when user distribution center coincides with cell center, the higher the user concentration, the larger the system capacity. And when user distribution center deviates from cell center, the loss of system capacity is much greater at higher user concentration.
\end{abstract}

Keywords- User Distribution; Max-C/I Scheduling; Round Robin Scheduling; Cellular Networks

\section{INTRODUCTION}

In a realistic cellular network, users are often not uniformly distributed as assumed by many previous studies [1]-[3]. There are hot spots where there are more users trying to get access to the wireless network such as shopping malls, train stations, office buildings, downtown area and etc. This clustered feature of user distribution will lead to a different system performance from the uniform distributed one.

Some studies have been done to analyze the effects of user distribution on system capacity [4], [5]. The user distribution model purposed in [4] separated a cell into two parts, a concentrated region near the base station and a uniform region near the cell boundary. Those two regions are controlled by several parameters such that when changing those parameters, the distribution could switch from uniform to semi-Gaussian. [5] purposed a symmetrical 2-D truncated Gaussian function. It defined a new variable: location distribution index. By changing this index, users can switch from base station centered to edge centered distribution.

In all these works, base station was always considered the center of user distribution. However, in this paper, we purpose a new user distribution pattern where both the user density and user distribution center can be adjusted by choosing proper parameters. It is more realistic because hot spots are not always coincided with base stations. Besides of user density, the location of base station is also depended on many other factors such as available infrastructures, costs, feasibilities and etc.

With our purposed user distribution model, we discuss its effects on system capacity under two most common used

\author{
Hongwen Yang \\ School of Information and Communication Engineering \\ Beijing University of Posts and Telecommunications
}

Beijing, China packet scheduling algorithms: Maximum carrier to interference (Max-C/I) scheduling algorithm and Round Robin (RR) scheduling algorithm. Their performances in forward and reverse links are both analyzed. We assume a single user being scheduled at a time and thus interference due to own-cell users is not considered.

The goal of this work is to answer the following question: how does the non-uniform user distribution influence system capacity? The main contribution of this work is to quantify numerically the system capacity with respect to a new user distribution.

The rest of this paper is organized as follows. Section II gives the purposed user distribution model and discusses its parameters. Section III gives the system model and analyzes the system capacity for forward link under two scheduling algorithms. Section IV analyzes the system capacity for reverse link. In section $\mathrm{V}$ numerical results are shown in terms of capacity (bps/Hz). Section VI concludes the paper.

\section{USER DistriBUTION MODEL}

We use a two-dimensional (2-D) truncated Gaussian function to model user probability density function. Assuming cell center is at the origin of the coordinates, for user $i$ with coordinate $\left(x_{i}, y_{i}\right)$ its location pdf is

$$
\begin{gathered}
f_{c}\left(x_{i}, y_{i}\right)=\beta \frac{\exp \left\{-\beta\left[\left(x_{i}-x_{0}\right)^{2}+\left(y_{i}-y_{0}\right)^{2}\right]\right\}}{\pi\left[1-\exp \left(-\beta \mathrm{R}_{\mathrm{c}}^{2}\right)\right]}, \\
\forall(x, y) \in\left\{x_{i}^{2}+y_{i}^{2} \leq R_{c}^{2}\right\}
\end{gathered}
$$

Where $\left(x_{0}, y_{0}\right)$ is the user distribution center, $R_{c}$ is the cell radius. As defined in [5], $\beta$ is the user distribution index and $\sigma$ is standard deviation for the 2-D Gaussian function. Notice that $\beta=0$ corresponds to uniform user distribution.

We now define an area index $\chi$ as

$$
\iint f_{c}\left(x_{i}, y_{i}\right) d\left(\chi \square \pi \mathrm{R}_{c}^{2}\right)=80 \%
$$

Which means $80 \%$ users concentrates within $\chi$ of the total cell region. $\chi$ gives a more direct description than $\beta$ to illustrate the influence of user distribution on system capacity. 


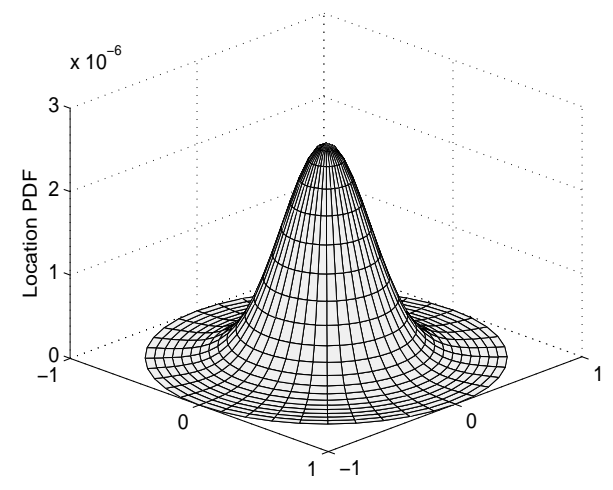

Figure 1. Symmetrical 2-D truncated Gaussian user distribution pdf over a cell region ( $\chi=0.2, x_{0}=y_{0}=0$, cell radius normalized to 1 )

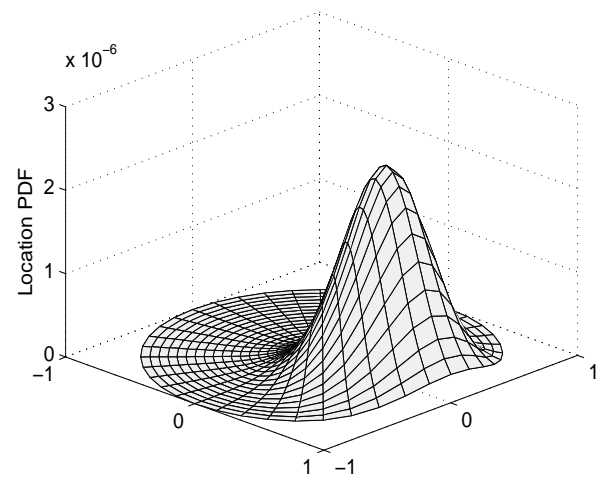

Figure 2. 2-D truncated Gaussian user distribution pdf over a cell region $\left(\chi=0.2, x_{0}=\mathrm{R}_{c} / 2, y_{0}=0\right.$ cell radius normalized to 1$)$

First considering that user distribution center coincides with its home cell center. That gives $x_{0}=y_{0}=0$. In this case users are distributed symmetrically over a cell region as an example shown in Fig.1.

When shifting user distribution center away from cell center, because users can only locate within a circle defined by $x_{i}^{2}+y_{i}^{2} \leq R_{c}^{2}$, user distribution is no longer symmetrical. For example, as shown in Fig.2 when shifting the distribution center along $x$ axis by $R_{c} / 2$, the resulting user distribution is concentrates on the $\left(R_{c} / 2,0\right)$ coordinate.

It is realistic to use a 2-D truncated Gaussian function modeling user distribution, in most of the case, users are not uniformly distributed within a cell, there often exist some hot spots. By shifting user distribution center, we can approximately model the hot spot locations in a cellular network.

\section{FORWARD LINK CAPACITY}

A typical layout of cellular network is considered as shown in Fig.3. Circular cell is used to approximate hexagonal cell.

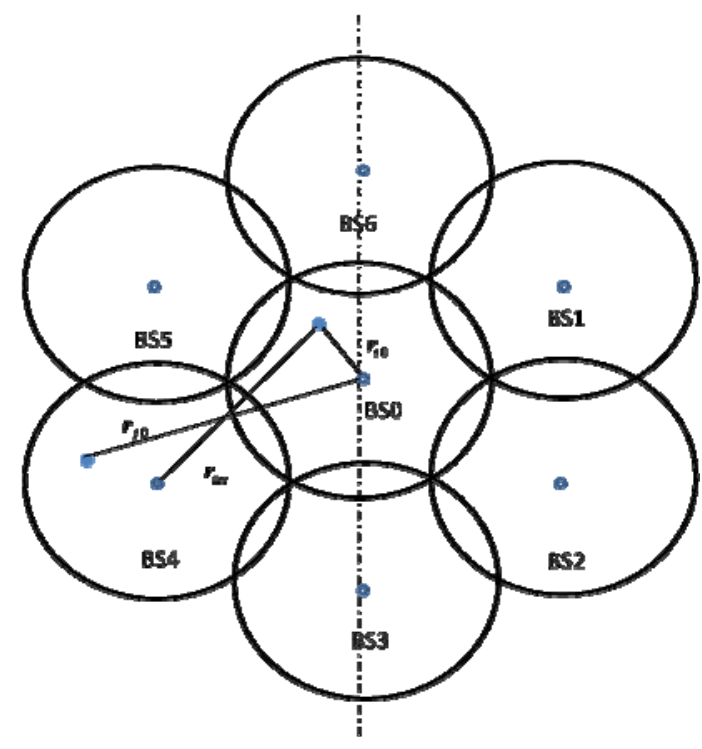

Figure 3. System Model

We assume cells sharing the same bandwith, but forward and reverse links are allocated in separated bands so that the interference between them is not considered. Assuming one user is scheduled at a time, we only consider interference from neighboring cells, interference from own cell is not considered thus.

The radio channel in our system is affected by both attenuation due to path loss and shadowing due to large scale fading. The link gain between mobile $i$ in home cell (BS0) and cell $m(\mathrm{BSm})$ is give by

$$
g_{i m}=r_{i m}^{-\alpha} 10^{\frac{\xi_{i m}}{10}}
$$

Where $r_{i m}$ is the distance between mobile $i$ in home cell and cell $m$. Supposing that the location of BS0 coincides with the origin of the coordinates as shown in Fig.3, ${ }^{r m}$ can be expressed as $r_{i m}=\sqrt{\left(x_{i}-x_{m}\right)^{2}+\left(y_{i}-y_{m}\right)^{2}}$, where $\left(x_{m}, y_{m}\right)$ is the coordinate of BSm. $\alpha$ is the path-loss exponent, ${ }_{i m}$ is a zero mean Gaussian random variable with deviation of $\sigma$ modeling the shadowing effect between mobile $\mathrm{i}$ and base station $\mathrm{m}$.

Assuming a single user being scheduled at a time, two typical packet scheduling algorithms are considered. They are Maximum carrier to interference (Max-C/I) scheduling and Round Robin (RR) scheduling.

\section{A. Forward Link Capacity with Max-C/I Scheduling}

For the forward link, each mobile $i$ in BS0 receives pilot signals from all the seven cells nearby. However, only the signal from its home cell is useful and signals from other six cells are considered interference. By measuring its signal to interference and noise ratio (SINR), it feedbacks this information to BS0, then BS0 will decide which mobile to schedule in the next instant. 
Max-C/I scheduling algorithm schedules the mobile with the instantaneously best radio link conditions i.e. the best SINR in the current time. Among the $N$ mobiles needed to be scheduled in BS0, the mobile $k$ chose by Max-C/I is [6]

$$
k=\underset{i=1}{\arg \max }\left\{\operatorname{SINR}_{i 0}^{D L}\right\}
$$

Where

$$
\operatorname{SINR}_{i 0}^{D L}=\frac{P g_{i 0}}{\mathrm{~N}_{0} \mathrm{~W}+\sum_{m=1}^{M} P g_{i m}}
$$

Then

$$
\operatorname{SINR}_{i 0}^{D L}=\frac{1}{\left(1 / \mathrm{SNR}_{i 0}\right)+\sum_{m=1}^{M}\left(g_{i m} / g_{i 0}\right)}
$$

With $M=6$ is the number of cells in the first ring and $P$ is transmission power of base station assuming each cell transmits at the same power level. Using (4), we can rewrite the first term in denominator as

$$
\mathrm{SNR}_{i 0}=\mathrm{SNR}_{\min }\left(\frac{R_{c}}{r_{i 0}}\right)^{\alpha} 10^{\frac{\xi_{i 0}}{10}}
$$

Where $\mathrm{SNR}_{\min }$ is the SNR for users at cell edge. Notice that $\mathrm{SNR}_{\min }$ makes user distribution transparent from the change of different cellular systems (CDMA,TDMA,FDMA, OFDMA...), it can be seen as an interface between user distribution and system parameters.

$$
C_{\text {Max-C/I }}^{D L}=\log _{2}\left(1+\operatorname{SINR}_{k 0}^{D L}\right)
$$

Users near cell center often have better channel conditions than edge users. When using Max-C/I scheduling algorithm, edge users will not be scheduled during a relatively long time and lead to starvation. Though lack of fairness, Max-C/I explores the multi-user diversity gain and therefore enjoys the upper bound of system throughput.

\section{B. Forward Link Capacity with Round Robin Scheduling}

As an alternative to the Max-C/I scheduling algorithm, Round Robin scheduling let the users take turns in using the shared resources, without taking the instantaneous channel conditions into account. The serving probability for each user in one cell is the same. Therefore RR algorithm achieves the best fairness while has a lowest system throughput.

For BS0 with $N$ users, each user shares $1 / N$ of the total system resource. For a TDM-base downlink as an example that will be $1 / N$ of total transmission time. Then the channel capacity for user $i$ is [7]

$$
C_{i}^{D L}=\frac{1}{N} \log _{2}\left(1+\operatorname{SINR}_{i 0}^{D L}\right)
$$

And the total system capacity is

$$
C_{R R}^{D L}=\sum_{n=1}^{N} C_{i}^{D L}
$$

\section{REVERSE LINK CAPACITY}

\section{A. Reverse Link Capacity with Max-C/I Scheduling}

For the reverse link, Max-C/I scheduling is a little different from that of forward link in that base station cannot measure the exact interference level for its serving users because it has no information about the scheduling condition of its neighbor cells. Users thus are scheduled based on their SNR level. If we use $i$ denote user in home cell, $j$ denote user in interference cells. Among the $N$ mobiles needed to be scheduled in BSm, the mobile $k^{(\mathrm{m})}$ chose by Max-C/I is

$$
k^{(\mathrm{m})}=\underset{j=1}{\arg \max }\left\{\mathrm{SNR}_{j 0}^{U L}\right\}
$$

Therefore

$$
\operatorname{SINR}_{k 0}^{U L}=\frac{1}{\left(1 / \mathrm{SNR}_{k 0}\right)+\sum_{m=1}^{M}\left(g_{k^{(\mathrm{m})} 0} / g_{k 0}\right)}
$$

The reverse link capacity is given by

$$
C_{\text {Max } C / 1}^{U L}=\log _{2}\left(1+\operatorname{SINR}_{k 0}^{U L}\right)
$$

Notice that since we only consider packet data transmission, uplink power control is not discussed here i.e. each user transmits at the same power level.

\section{B. Reverse Link Capacity with Round Robin Scheduling}

Round Robin scheduling algorithm in the reverse link is almost the same as in the forward link. Each user has the same priority to be served by its home cell. And this gives

$$
C_{i}^{U L}=\frac{1}{N} \log _{2}\left(1+\operatorname{SINR}_{i 0}^{U L}\right)
$$

Where

$$
\operatorname{SINR}_{i 0}^{U L}=\frac{1}{\left(1 / \mathrm{SNR}_{i 0}\right)+\sum_{m=1}^{M}\left(g_{j 0} / g_{i 0}\right)}
$$

And the total system capacity is

$$
C_{R R}^{U L}=\sum_{n=1}^{N} C_{i}^{U L}
$$

User $i$ and User $j$ are chosen by turn from BS0 and BSm respectively.

\section{NUMERICAL RESULTS}

In this section, curves are presented showing the results obtained for system capacity in terms of bps/Hz. Simulation is carried out on Max-C/I and RR scheduling for both forward and reverse links. For all the following figures the parameters considered by default are: $\mathrm{SNR}_{\min }=34 d B$ [8], a path loss exponent of 4 and a shadowing deviation of $8 \mathrm{~dB}$ [1].

We first explore the influence of user area index $\chi$ on system capacity. Assuming user distribution center coincides 


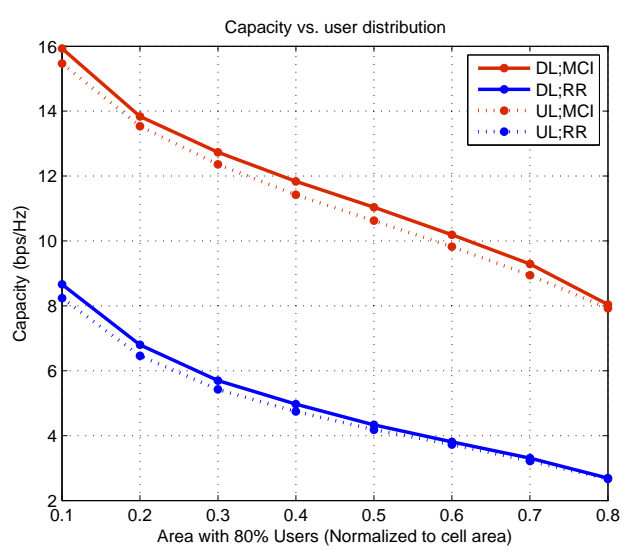

Figure 4. System capacity with respect to the area index $\$ \backslash$ chi\$

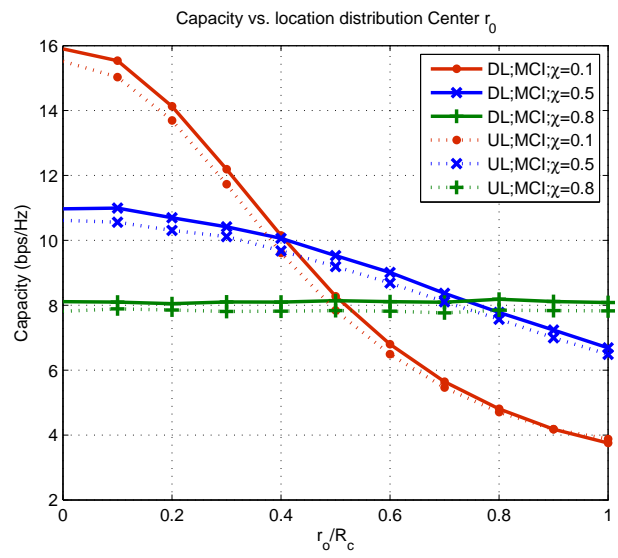

Figure 5. System capacity with respect to the user distribution center $\$ r \_\$$ for Max-C/I scheduling

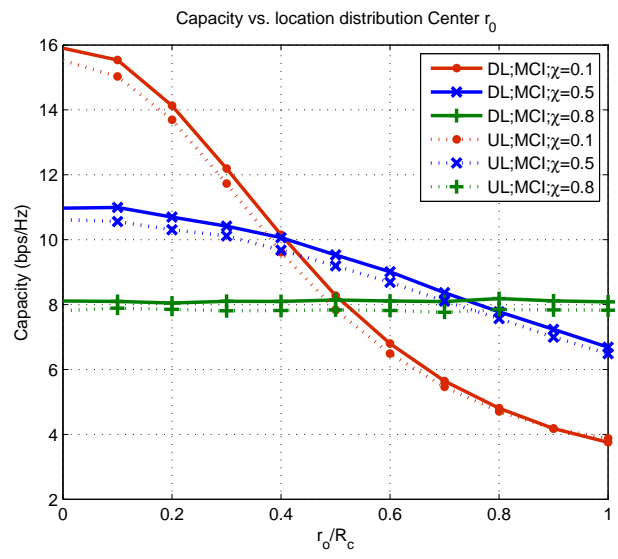

Figure 6. System capacity with respect to the user distribution center $\$ r \_\$$ for Round Robin scheduling

with cell center, for Max-C/I scheduling and RR scheduling in both forward and reverse links, system capacity decreases as user becomes less concentration i.e. from $80 \%$ users concentrate within $10 \%$ area to $80 \%$ users concentrate within $80 \%$ area as shown in Fig.4. For Max-C/I scheduling, its capacity drops $50 \%$ and for RR scheduling, it drops nearly $75 \%$. Also notice that Max-C/I scheduling always has a better performance than RR scheduling in terms of system capacity.

The effect of user center location on system capacity is showed in Fig.5 and Fig.6 for Max-C/I and RR scheduling respectively. As user distribution center deviates from cell center $r_{0} / R_{c}=0$ to cell edge $r_{0} / R_{c}=1$ where $r_{0}^{2}=x_{0}^{2}+y_{0}^{2}$, system capacity keeps decreasing and the smaller the area index $\chi$, the larger the capacity decreases except at $\chi=0.8$ which corresponding to an uniform user distribution, where the deviation of user center does not influence system capacity. Notice also that capacity at high user concentration will be less than the uniform case when user center shifts to a certain location.

\section{CONCLUSION}

In this paper, we propose a non-uniform user probability density function and introduce a more realistic parameter: area index, to indicate the degree of user concentration. Its effects on system capacity are addressed for both forward and reverse links under Max-C/I and Round Robin scheduling algorithms. Our results show that when user distribution center is at the cell center, cellular network always benefits from a concentrated user distribution. The higher the concentration i.e. the smaller the $\chi$, the larger the system capacity. When shifting user distribution center, the loss of system capacity is much greater at higher user concentration. User center can be used to model hot spots in a cellular network. Future work will address the situation of multiple user centers (hot spots) within a cell.

\section{REFERENCES}

[1] R. K.S. Gilhousen, I.M. Jacobs, "On the capacity of a cellular cdma system," IEEE Transaction on Vehicular Technology, vol. 40, no. 2, pp. 303-312, May 1991.

[2] C.-L. J.M. Romero-Jerez and A. Diaz-Estrella, "Effect of power control imperfections on the reverse link of cellular cdma networks under multipath fading," IEEE Transaction on Vehicular Technology, vol. 53, no. 1, pp. 61-71, Jan. 2004.

[3] W.M. Tan and F.C.M. Lau, "Analysis of power control and its imperfections in cdma cellular systems," IEEE Transaction on Vehicular Technology, vol. 48, no. 5, pp. 1706-1717, Sep. 1999.

[4] M. Elalem and L. Zhao, "Realistic user distribution and its impact on capacity and coverage for a wcdma mobile network," Wireless Personal Commun., pp. 1-5, 2009.

[5] D. Zhuye Lei and N.B.Mandayam, "Location-dependent other-cell interference and its effect on the uplink capacity of a cellular cdma system," IEEE VTC’99., vol. 3, pp. 2164-2168, 1999.

[6] H. Fu and D. I. Kim, "Analysis of throughput and fairness with downlink scheduling in wcdma networks," IEEE Transaction on wireless communications, vol. 5, no. 8, pp. 2164-2174, Aug. 2006.

[7] S.-L. Kim and H.-M. Baek, "On the economic aspects of downlink scheduling in ds-cdma systems: Base-station density perspective," IEEE Transaction on Vehicular Technology, vol. 55, no. 5, pp. 1594-1602, Sep. 2006.

[8] C. C.Chevallier and A.Garavaglia, WCDMS (UMTS) Deployment Handbook Planning and Optimization Aspects. Wiley, 2006. 